\title{
CEREBELLAR SYNDROME IN AN ADULT WITH MAL- FORMATION OF THE CEREBELLUM AND BRAIN STEM (ARNOLD-CHIARI DEFORMITY), WITH A NOTE ON THE OCCURRENCE OF “TORPEDOES" IN THE CEREBELLUM
}

\author{
BY \\ C. D. ARING \\ From the Pathological Laboratory, National Hospital, Queen Square, London
}

(Received 14th December, 1937)

OBSERVATIONS are plentiful on congenital malformations of the nervous system which cause symptoms. The great majority of these studies, however, have been made in infants dying shortly after birth. It is of some interest to record a case of malformation of the cerebellum and brain stem, apparently congenital, which did not cause symptoms of a disabling nature until adulthood. This is the purpose of this paper. It is thought also that by calling attention to the histological changes in the cerebellum of this case that such changes may be removed from the category of the uncommon. It is possible that if careful search were made in the cerebellum of patients succumbing to the various " cerebellar system" diseases, structural changes of a similar kind would be found more often than is the case.

\section{Case Report}

J.P., a wood-worker, aged 20, was admitted to the National Hospital, Queen Square, on 30th August, 1935, under the late Dr. S. A. Kinnier Wilson.

The patient had always been well until 18 months before admission. At that time he noticed slight unsteadiness of the lower extremities, had difficulty in keeping his balance, and began to stagger on walking. There was a gradual increase in the severity of these symptoms and the progress of the disease during 8 months before admission. Three months before admission he had had bouts of sneezing, and at that time he could not swallow properly, for on several occasions fluid came back through his nose. There had, however, been no recurrence of these symptoms. At the time of admission to the National Hospital he said that he had difficulty in standing unsupported. Both legs were equally affected and he would stagger or fall to either side. He did not think the upper extremities were involved. There had never been weakness of any of the extremities. The patient had suffered from dull, frontal headaches for as long as he could remember, but they had not been incapacitating. The past history contained nothing indicating previous involvement of the nervous system or any incident that could be directly related to the condition of the patient at 
the time of entrance to the hospital. The history obtained from the patient and from the parents revealed no instance of nervous or mental disease in any of his family.

On examination he was found to be somewhat slow mentally and he co-operated poorly. His speech was rather slow and slurred. There was a coarse horizontal nystagmus on looking upward or to the right, while on looking to the left the nystagmus was more rapid and not so coarse. The left side of the palate arched on phonation rather more than the right, indicating a slight weakness of the right side of the palate. The right sternomastoid and trapezius muscles were not so well developed or as powerful as those of the left. There was a fascicular tremor throughout the tongue musculature. The head was tilted slightly toward the right shoulder and there was a fine tremor of the head. Apart from these findings the functions of the cranial nerves and the special senses were normal. A pes equino-cavus deformity of both feet was noted. No alteration of development or motor power of the extremities was observed, but there was a generalized increase in muscle tone, more obvious in the lower extremities. A coarse fascicular tremor was present in the muscles of the left extremities. There was a fine tremor of the outstretched hands, and finger to nose tests were performed with a moderate terminal tremor. Rapid alternating movements of the hands were slow and " toe-wiggling" was poorly performed. Placing the heel on the knee, then directing the great toe towards the examiner's finger, was performed with moderate ataxia bilaterally. It was evident that spasticity and ataxia rather than weakness were the cause of the disability. The gait was very ataxic ; the patient walked slowly and stiffly on a wide base and with his gaze fixed on the ground. Closure of the eyes greatly increased the unsteadiness. All of the tendon reflexes were very brisk; knee and ankle clonus was obtained bilaterally. The abdominal reflexes were normal and the plantar responses were extensor in type. The patient occasionally failed to identify passive toe movements, otherwise there was no sensory abnormality. The general physical examination was entirely normal.

A lumbar puncture performed on 31st, August 1935, gave a clear fluid under normal pressure. There were 2 cells per $\mathrm{cm}$., a total protein of 0.22 per cent., and strongly positive globulin reactions. Lange's gold sol test was normal and the Wassermann reaction was negative in both the cerebrospinal fluid and the blood.

The patient was at first thought to be suffering from spino-cerebellar ataxia. He commenced to exhibit a peculiar mental reaction, becoming morose and occasionally very belligerent and abusive. The nystagmus continued to be slower and coarser on looking to the right. This fact, together with the high protein content of the cerebrospinal fluid, suggested the possibility of a right cerebellar cyst. On 13th November, 1935, a bilateral ventricular tap was performed and the withdrawn fluid replaced by air. The ventricular fluids were entirely normal and contained 0.01 per cent. of protein. The ventriculograms were also normal. On 3rd December, 1935, a cerebellar exploration revealed nothing of note besides marked herniation through the foramen magnum of the tonsils of the cerebellum, which were attached tightly together by adhesions in the arachnoid. There was no increase of intracranial pressure. The arch of the atlas was removed in order to liberate the tonsils, which even then were difficult to mobilize, as they reached down to the axis. The right cerebellar tonsil was somewhat larger than the left and the vermis was displaced slightly toward the left. The arachnoid at the lower end of the vermis and between the tonsils was considerably thickened. The adhesions between the tonsils were broken down with difficulty, after which the lower end of the fourth ventricle and the medulla could be seen. Exploration of the cerebellar lobes revealed no tumour. The patient recovered consciousness shortly after the operation had been completed and recognized individuals. Several hours later the pulse quickened and he developed Cheyne-Stokes respiration and cyanosis. His temperature was subnormal. He gradually became worse, the pulse becoming feeble and more rapid, and death occurred on 4th December, 1935, eighteen hours after operation. 


\section{Pathological Findings}

A necropsy was performed two hours after death and the brain and cord were removed. Several abnormalities were visible to the naked eye. Both tonsils of the cerebellum passed downward over the dorsal surface of the medulla, to which they were firmly attached. The appearance was not that of a cerebellar pressure cone, as the right tonsil lay unusually far dorsally and seemed to be somewhat enlarged. The dome of the cerebellum lay asymmetrically, the highest point being to the left of the midline. The most rostral portion of the medulla appeared unusually thin and soft, so that the brain stem tended to bend abnormally at this level. No cause for death and no hæmorrhage at the site of operation were found.

Pieces from each spinal segment, from various levels of the brain stem, and from different parts of the cerebellum and cerebral cortex were examined by several histological methods. The Weil, Weigert-Pal and Mallory's phosphotungstic acid hæmatoxylin methods were applied to the brain stem and to the spinal cord. A moderate degree of degeneration was present in the anterior and posterior roots, especially in the cervical region. These stains revealed definite demyelinization in the cord affecting rather diffusely the anterolateral tracts. In the cervical region the dorsal and possibly also the ventral spinocerebellar tracts were spared to some extent. There was some demyelinization and fairly intense neuroglial sclerosis in the columns of Goll from the third cervical segment upward and slighter neurolgial overgrowth at lower levels of the cervical cord. There was some distension of the central canal of the cord at the levels of cervical segments six, seven, and eight. This was lined only partly on its ventral surface by ependyma and reached but did not penetrate the base of the dorsal horn on either side. The ventral portion of the medulla was shrunken and distorted throughout. At the caudal level (Fig. 1, E) the medulla was flattened in its antero-posterior diameter despite the very obvious prominence of the dorsal columns. The column of Goll projected dorsally outwards from the columns of Burdach, resulting in an abnormal outline of the medulla at this level. At a slightly higher level (Fig. 1, D) there was a rather deep indentation of the white matter of the medulla just ventral to the nucleus of the spinal root of the trigeminal nerve, which dislocated the dorsal spino-cerebellar tract somewhat in a ventral and medial direction. At a level about half-way up the medulla the olives and restiform bodies were greatly shrunken, so that the whole transverse diameter of the medulla was abruptly reduced and the width on cross-section was greatly narrowed. The cells of the olives in this area were shrunken and stained darkly and were very irregularly spaced in the foliæ as though many had disappeared. There was considerable gliosis in the hilum of the olives. The olives although much shrunken remained visible up to the junction of the medulla and pons. At about the level of the middle of the inferior olives (Fig. 1, C) one observed besides the distortion and shrinkage that the calamus scriptorius was overhung more on one side than the other by the inferior medullary velum and a cleft was formed to one side, running a short distance into the grey matter of the floor of the ventricle in this region. 
At the upper level of the medulla (Fig. 1, B) there was comparatively little distortion besides pinching of the ventrolateral margins, but there was obvious reduction in the size of all structures in this area. The pons as a whole was shrunken, especially in its caudal two-thirds. The pinching of the ventrolateral margin was very obvious in the caudal part of the pons and the transverse fibres were very pale in the caudal one-third of the pons.

Various staining methods (Mallory's phosphotungstic acid hæmatoxylin, hæmatoxylin and van Gieson, silver counterstained with thionin) applied to the cerebellum revealed no abnormality. There was no dropping out of Purkinje cells and no evident loss of fibres; the roof nuclei and other structures in the cerebellum appeared entirely normal. However, when the Gros method for silver impregnation was utilized and the reduction in ammonium silver nitrate allowed to proceed until the axis cylinders of the Purkinje cells had taken the stain fully (to the detriment of the medullary parts of the cerebellum), it was seen that practically all of the individual axones of the Purkinje cells contained a bulbous swelling sometimes called a "torpedo" (Fig. 2). Under high magnification the fibres of the baskets surrounding the Purkinje cells could be followed into the granular layer as they travelled along the Purkinje axones to envelop the "torpedoes." These bulbous swellings of the Purkinje axones were generalized throughout the cerebellar folia in the same profusion as illustrated in the photograph. No abnormality was found in the cerebral cortex.

In summary a man aged 20 began to complain of unsteadiness in the lower extremities 22 months before death. There was gradual progression until 18 months later, when he had difficulty in standing unsupported and the gait was staggering in type. Examination 4 months before death revealed mental changes, nystagmus, and hesitant speech. There was increased muscle tone in all of the extremities. The gait was very ataxic. The tendon reflexes were extremely brisk and the plantar responses were extensor in type. Sensation was practically intact. The cerebrospinal fluid was normal except for a great excess of protein content. His status remained unchanged for nearly four months. Death occurred after cerebellar exploration. Necropsy revealed adhesions between the elongated cerebellar tonsils, both of which were adherent to the medulla. The medulla was small and deformed, chiefly because of " wasting" in the region of the olives and of the olives themselves in the rostral half of the medulla. The caudal part of the pons was shrunken and there was a loss of transverse fibres in the pons. The pyramidal tracts and spino-cerebellar tracts in the cord were somewhat degenerated in the lower levels and approximated to the normal in the higher sections of the cervical cord and medulla. There was moderate degeneration in the columns of Goll. In the cerebellum numerous " torpedoes" in the axones of the Purkinje cells were found. 


\section{Discussion}

As the case appears to represent several of the characteristics found in the Arnold-Chiari deformity (Arnold (1894), Chiari (1891, 1896), Parker and McConnell (1937), Russell and Donald (1935), Schwalbe and Gredig (1907)), it will be helpful to outline the several findings which have led in the past to a diagnosis of this deformity.

The Arnold-Chiari deformity has been described in a fœtus and in a subject of 68 years of age. Clinical symptoms and signs recorded in the literature include those commonly found in association with disturbance of pyramidal function and with hydrocephalus. It has not been possible to trace any record of a case presenting symptoms and signs of a cerebellar syndrome. Through the kindness of Coburn and Penfield (1938) I have been informed of a case which they are reporting elsewhere. This is a case of a female patient aged 29 years. During childhood she had an operation for the repair of a thoracic spina bifida. Later she developed a tendency to fall forward occasionally. This, along with a horizontal and vertical nystagmus, suggested some cerebellar abnormality, for which she was explored. Two months later she died.

Pathologically the most obvious abnormality of the nervous system with the Arnold-Chiari deformity is an elongation of the tonsils and inferior portion of the cerebellum, so that these parts may extend downward for varying distances into the spinal canal. The pia arachnoid covering this malformation is usually thickened and may cause the prolongations of cerebellum to be adherent to the brain stem. Hydrocephalus and spina bifida are usually but not always present. The pons and medulla are commonly noted to be flattened or bent or asymmetrical and occasionally lack the ventral protuberance. Hypoplasia of the cerebellum and pons have been observed in cases of the Arnold-Chiari deformity. Hydromyelia of the cord has sometimes been found. No very satisfactory microscopic study of the nervous system has been performed to date. Examination of the elongation of the cerebellum has revealed sclerosis in some cases and normal cerebellar convolutions in others.

The pathology of the present case corresponds in a general way to that which has been previously described for the Arnold-Chiari deformity. The elongations of the cerebellum and the cohesive pia arachnoid found at the operation and again at autopsy are rather typical. The small pons and deformed medulla found in this case are in keeping, and shrunken olives have been noted in Chiari's (1891) descriptions. In some of his cases Chiari recorded abnormalities of the olives, which ranged from "olives placed with their longitudinal axis oblique" to complete absence of the olives in one case. There was no spina bifida in this case, but no special search was made for it ; nor was there any hydrocephalus.

The possibility that this case might represent one of the variants of spinocerebellar ataxia (Chart 1) must be considered. " Hereditary cerebellar ataxia," the title which Marie (1893) advanced for the cases with primary involvement of the cerebellum, was the result of the collation of the cases of other authors. As Holmes (1907) has pointed out, the cases collected by Marie for inclusion under this title were dispersed by anatomical examination of the nervous systems of 
these individuals. Despite this fact, the heading " Marie's hereditary cerebellar ataxia " remains a common one. It is well to dispense with the term and to list the cases where they belong, either under the heading of primary parenchymatous degeneration of the cerebellum (Holmes, 1907) or olivo-ponto-cerebellar atrophy. Of the various forms of spino-cerebellar degeneration this case most nearly resembles olivo-ponto-cerebellar atrophy. The age of onset in olivo-pontocerebellar atrophy commonly commences late in life, usually in the sixth decade. Mental symptoms are not uncommon in this form, and except for these features it is doubtful if any clinical differentiation is possible. In olivo-ponto-cerebellar degeneration the inferior olive and middle peduncle are the structures most frequently and severely involved, as they were in this case. Degeneration of the long tracts of the spinal cord has been recorded in olivo-ponto-cerebellar atrophy. In the present case the diffuse degeneration of the antero-lateral tracts and the degeneration of the posterior columns of Goll in the upper cervical region were of sufficient severity to cause clinical signs.

Chart 1

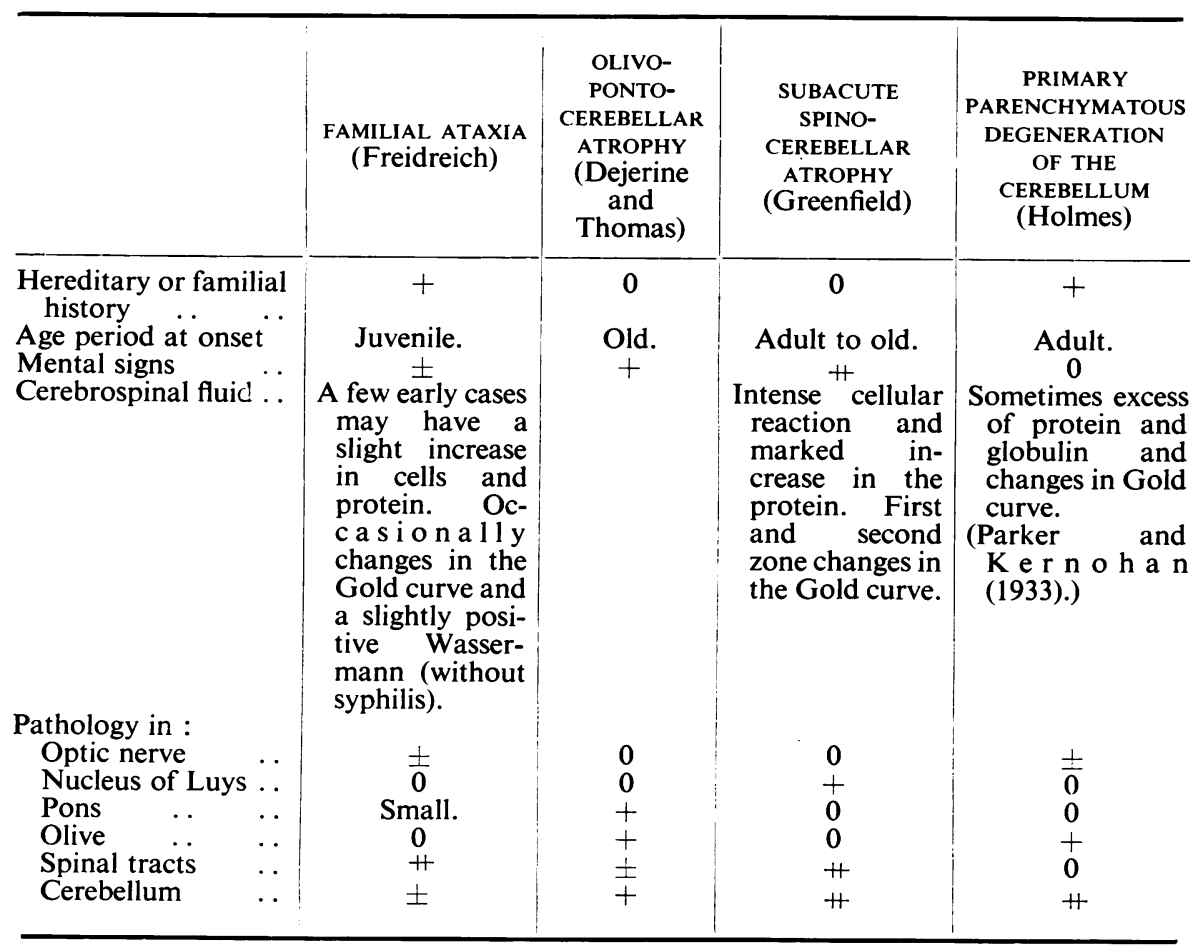

+ , usually present.

0 , usually absent. \pm , may or may not be present. \#, severe involvement.

Although the white matter of the cerebellum showed no evident loss of fibres the presence of "torpedoes" indicates that this was beginning. The occurrence of numerous " torpedoes" in the cerebellum is never a common 
finding and was one of the most striking features in the pathology of this case. These swellings of the axones of the Purkinje cells were the only pathological findings in the cerebellum. In olivo-ponto-cerebellar atrophy as described by Dejerine and Thomas (1900), the cortex of the cerebellum atrophies. However, cases have been reported in which the inferior olive and nuclei pontis were atrophied without any evident degeneration of the cerebellum and others in which the olives were degenerated with little or no degeneration of the pontine nuclei or middle peduncles and with involvement of the dentate nuclei.

Olivo-ponto-cerebellar atrophy is probably the commonest and most clearcut entity of the spino-cerebellar ataxias, but even in this sub-heading (Chart 1) many intermediate and transitional states exist, one of which might be represented by this case, which it would be difficult to classify with any of the other well-known types of spino-cerebellar degeneration. A glance at Chart 1 will readily corroborate this statement and accordingly curtail the discussion. The rapid onset of cerebellar symptoms in early adult life in this case is indeed an unusual phenomenon, for which the cause remains unexplained. It would appear to be comparable with the onset in Coburn and Penfield's case (aged 29 years) and with the onset of hydrocephalic symptoms in Parker and McConnell's cases (aged 10, 18, and 32 years) of the Arnold-Chiari deformity.

\section{"Torpedoes" in the Cerebellum}

Sections from the cerebellum of neurological cases selected at random were subjected to the Gros method for silver impregnation. "Torpedoes" were found in moderate numbers in two cases of olive-ponto-cerebellar atrophy and in a case of granular ependymitis. In the former there was a great loss of Purkinje cells and the baskets appeared disintegrated. Where a " torpedo" was found one could trace the axone centrifugally up through the basket fibres to a Purkinje cell. In the case of granular ependymitis there was no loss of Purkinje cells and the cerebellum appeared otherwise intact. In none of these cases did the "torpedoes" appear in anywhere near the numbers seen in the cerebellum of the case reported in detail (Fig. 2). Rare "torpedoes" were found in the cerebella of individual patients succumbing to dementia paralytica (Fig. 3), syphilis of the central nervous system with granular ependymitis and atrophy of the left cerebral hemisphere with convulsive seizures, and of two patients with amaurotic family idiocy (Tay-Sachs). In this group of cases it was usually found that the Purkinje cell whose axone was involved exhibited no visible abnormality. No "torpedoes" were found in the cerebella of the following single cases : syphilitic myelitis with syringomyelia, tabes dorsalis, neuromyelitis optica, post-encephalitic Parkinsonism, acute chorea, disseminate sclerosis, old cerebral contusion, amaurotic family idiocy (Bielschowsky type), cerebellar abscess, and cerebellar agenesis. Neither were they seen in four cases of dementia paralytica.

This round or spindle-shaped and usually homogeneous swelling incorporated along the course of the axone of the Purkinje cell has been noted by other workers in cases of dementia paralytica, in diffuse atrophies and localized 
disease of the cerebellum, in tuberous sclerosis, dementia præcox, familial ataxia (Friedreich), amaurotic family idiocy, and in olivo-ponto-cerebellar atrophy. Bielschowsky (1920) looked upon this axone swelling as a "trivial reaction phenomenon " and Spielmeyer (1922) noted that this swelling might be a primary injury of the axis cylinder due to all sorts of acute and chronic causes.

This change is present in varying degree in cerebella where no other abnormality is found and in cerebella with other marked anatomical changes. An attempt to connect this individual pathological change with neurological dysfunction would be speculative. I have been unable to find the report of a case of swelling in the axis cylinders of the Purkinje cells of the cerebellum without change elsewhere in the central nervous system.

\section{Summary}

A case is described of a man aged 20, who died 22 months after the onset of symptoms and who presented many of the pathological findings of the ArnoldChiari deformity. The history was one of progressive ataxia of the lower extremities. Mental changes, nystagmus, hyperactive tendon reflexes, and extensor plantar responses were also present. There was an excess of protein in the cerebrospinal fluid.

Pathologically the following abnormalities were found : a small deformed medulla with degeneration of the olives in the rostral one-half of the medulla, reduction in the size of the pons with degeneration of the transverse fibres, especially those in the caudal one-third of the pons, degeneration of the spinocerebellar and pyramidal tracts in the lower levels of the spinal cord, and moderate degeneration in the columns of Goll and in the anterior and posterior roots of the upper segments of the cervical cord. The cerebellum was intact except for the presence of a bulbous swelling (" torpedoes") in a great many of the axones of the Purkinje cells.

The presence of "torpedoes" in moderate numbers in the axones of Purkinje cells was found in cases of olivo-ponto-cerebellar atrophy and granular ependymitis. Occasional "torpedoes" were observed in cases of dementia paralytica, syphilis of the central nervous system, atrophy of one cerebra hemisphere, and amaurotic family idiocy (Tay-Sachs).

I am indebted to Dr. J. G. Greenfield for his helpful guidance. This work was carried out during the tenure of a Rockefeller Fellowship.

\section{REFERENCES}

Arnold, J. (1894). Beitr. path. Anat. (Jena), 16, 1.

Bielschowsky, M. (1920). J. Psychol. Neurol., 26, 123.

Chiari, H. (1891). Dtsch. med. Wsch., 17, 1,172.

- (1896). Denksch. Akad. Wiss. Wien (Mathemat. Naturwiss. Classe), 63, 71.

Coburn, D., and Penfield, W. (1938). Arch. Neurol. Psychiat. Chicago.' (In press.)

Dejerine, A., and Thomas, A. (1900). Novv. Iconogr. Salpêt, 8, 330.

Holmes, G. (1907). Brain, 30, 466 . (1907). Brain, 30, 545.

Marie, P. (1893). Sem. méd. Paris, 8, 444.

Parker, H. L., and Kernohan, J. W. (1933). Brain, 56, 191.

and McConnell, A. A. (1937). Trans. Amer. neurol. Ass.

Russell, D. S., and Donald, C. (1935). Brain, 58, 203.

Schwalbe, E., and Gredig, M. (1907). Beitr. path. Anat. (Jena), 40, 132.

Spielmeyer, W. (1922). Histopathologie des Nervensystems. Springer, Berlin. 

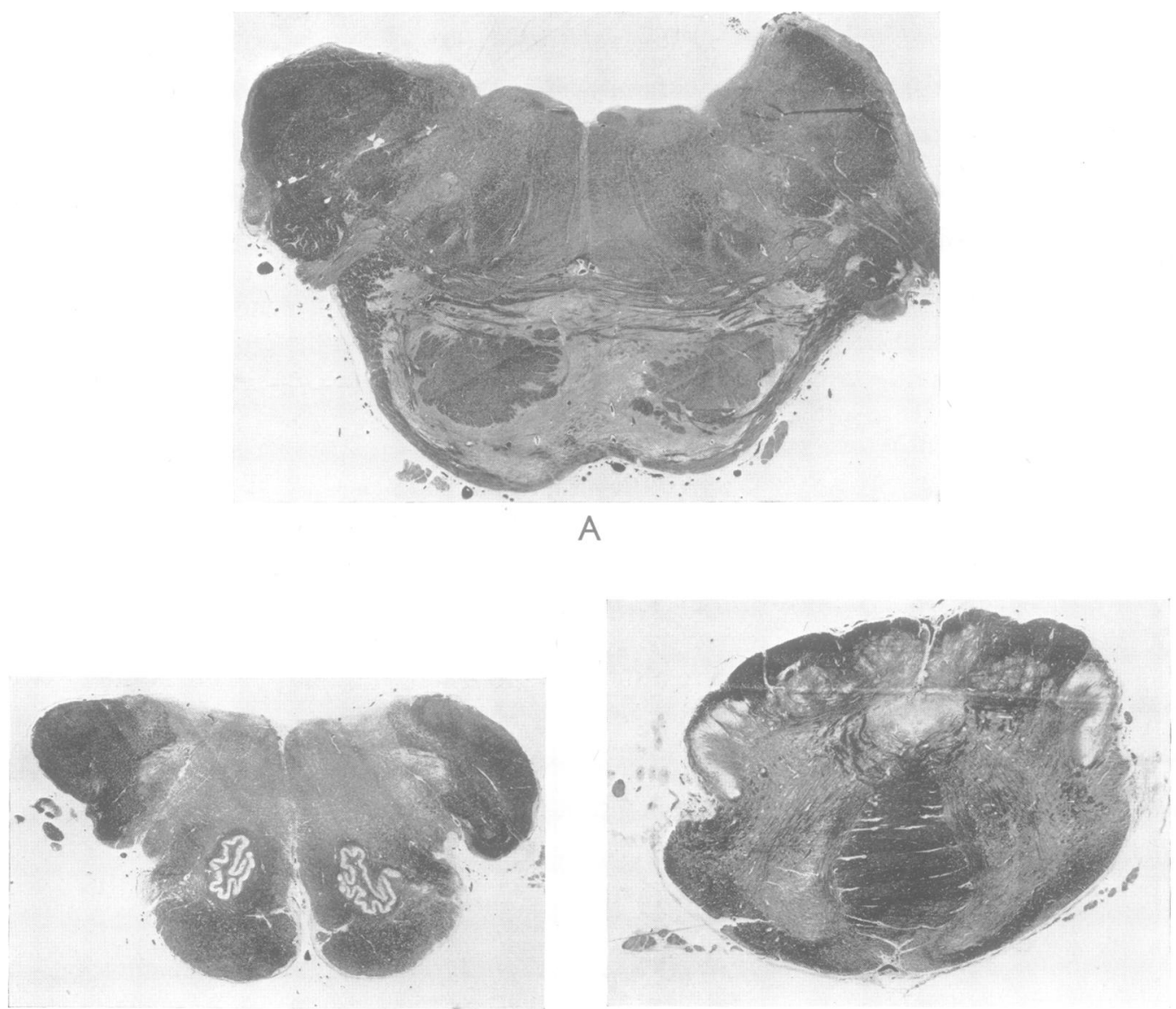

B

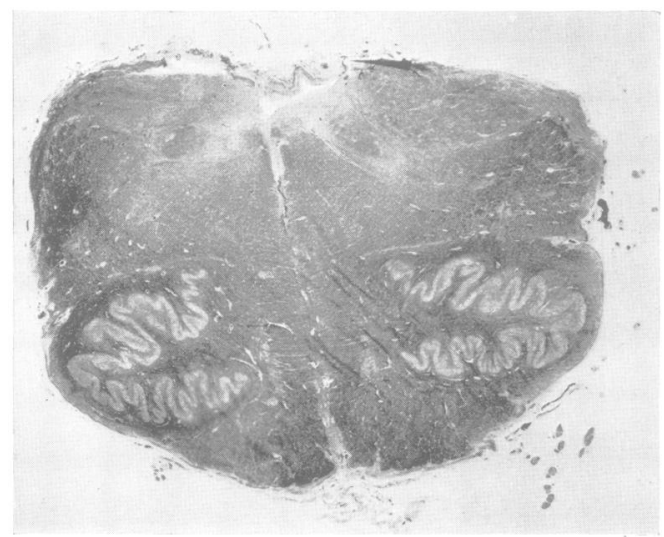

C

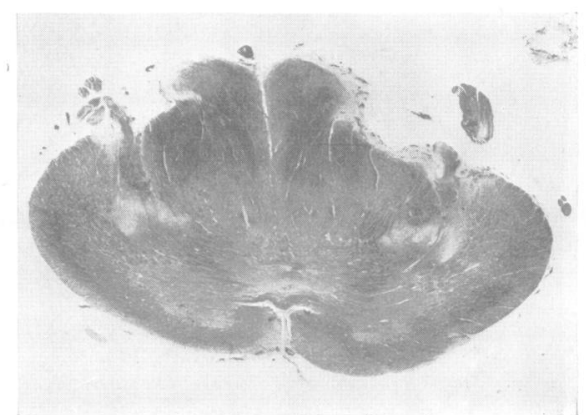

$E$

Fig. 1.-Sections from various levels of the brain stem to illustrate the distortion and shrinkage of the ventral half of the medulla. Weil method for myelin sheaths. Magnification the same throughout this series : $\times 6 \cdot 75$. 


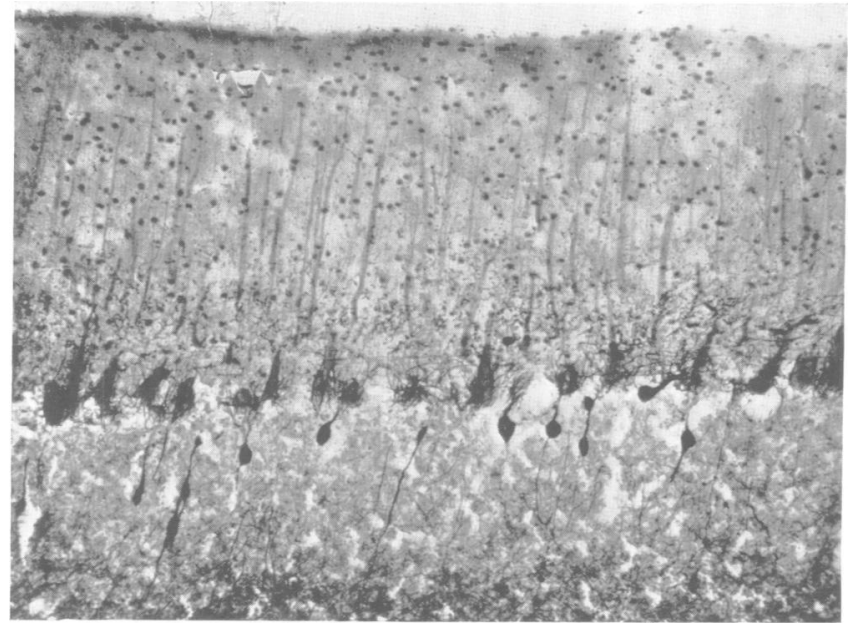

Fig. 2.-A photomicrograph indicating the profusion of "torpedoes" in the axones of the Purkinje cells of the cerebellum in the case of Arnold-Chiari deformity of the brain stem described in the text. Gros method for silver impregnation.

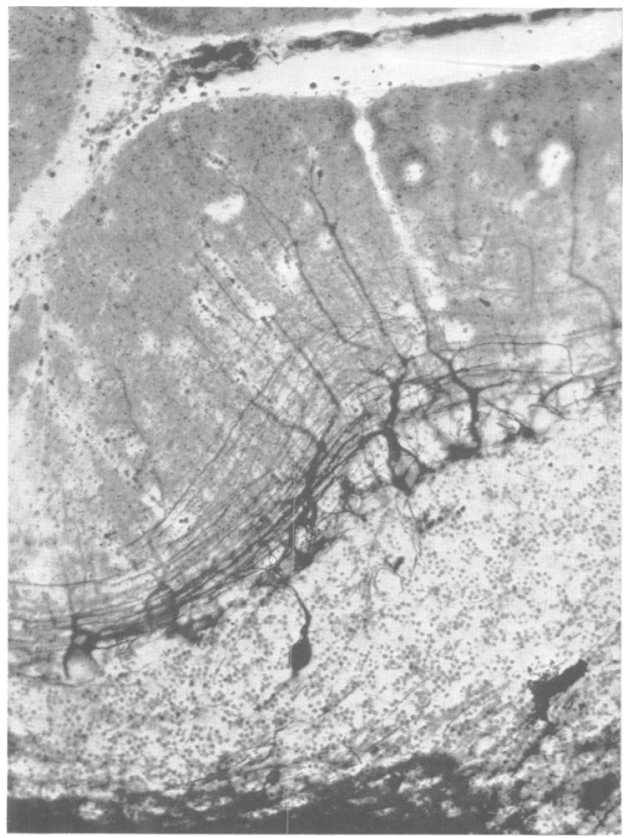

Fig. 3.-A " torpedo" in the cerebellum of a case of dementia paralytica. Gros method for silver impregnation. 